\title{
SIMULATED ANNEALING ALGORITHM FOR OPTIMIZING MULTI-PROJECT LINEAR SCHEDULING WITH MULTIPLE RESOURCE CONSTRAINTS
}

\author{
Po-Han Chen \\ Assistant Professor \\ School of Civil \& Environmental Engineering, \\ Nanyang Technological University, Singapore \\ cphchen@ntu.edu.sg
}

\author{
Seyed Mohsen Shahandashti \\ Research Student \\ School of Civil \& Environmental Engineering, \\ Nanyang Technological University, Singapore \\ Seye0001@ntu.edu.sg
}

\begin{abstract}
This paper integrates multi-project scheduling and linear scheduling concepts. Since the problem is combinatorial, a two-stage heuristic solution-finding procedure is used to model the problem with multiple resource constraints. Simulated annealing is utilized as a searching engine in the second stage to find the probable optimized solution. The first stage is slightly different from the other two-stage solution finding procedures which are proposed till now. A numerical example of a multi-project situation is given and solved as well.
\end{abstract}

\section{KEYWORDS}

Linear Scheduling, Multi Projects, Multiple Resource Constraints, Simulated Annealing.

\section{INTRODUCTION}

Since CPM is not effective for scheduling of linear projects, some other methods like Line of Balance method (LOB) [1], Vertical Production Method (VPM) [2] and Linear Scheduling Method (LSM) [3] have been developed. LSM is utilized in this research. Practically, resources are limited in construction projects so multiple resource constraints are incorporated in the model by" resource leveling" and "resource allocation". Optimizing linear scheduling projects with multiple resource constraints is a combinatorial optimization problem, so it should be optimized by one of the combinatorial optimization tools. Simulated annealing is chosen for achieving this goal in this paper.

LSM was introduced by Johnston in 1981 [3] in a highway construction project. Figure 1 depicts a Linear Scheduling Method diagram. It is a time versus location or distance diagram. Activities are presented as line segments, blocks, or bars in the diagram. The slop of the line segments represents the production rate of the corresponding activities
[4]. Harmelink (1995) [5] established a heuristic algorithm to determine the controlling activities path but without consideration of resource limitations. "Controlling activity path" in linear scheduling is a concept similar to "critical path" in CPM. Controlling activity path is a set of activities that constitute a path and dictate project duration. Mattila (1997) [6] considered resource leveling in a model of highway construction project. He solved the model by using mixed integer programming. Liu (1999) [7] considered single resource allocation and proposed a heuristic solution procedure using the Tabu Search algorithm in his model. The model included two stages. Lue \& Hwang (2001) [8] proposed a precast production project and solved it with a genetic algorithm-based model.

Simulated annealing is introduced in 1983 in the science magazine [9] in 1983.This concept has been used in several engineering applications since 1983 but it is introduced to construction management by Chung-I Yen (2005) [10]. He proposed simulated annealing for optimizing linear scheduling projects with multiple resource 
constraints. He considered resource allocation and resource leveling simultaneously.

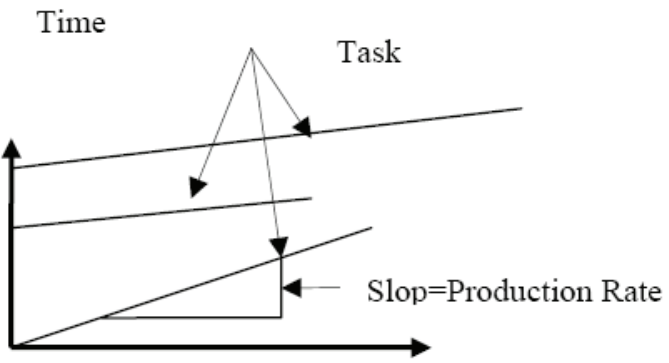

Locations

Figure 1 Typical Linear Scheduling Diagram in Highway Construction

\section{MULTI PROJECTS}

The resource-constrained multi-project problem is defined as one in which two or more projects are concurrently active [11]. There are two approaches for scheduling of multi-project situations i.e. multi-project and single project approach. In the former, projects are considered separately but projects are connected to each other in the latter. The multi-project approach is used in this paper. The first attempt in this field is done by Fendley (1968) and a lot of heuristics are proposed since 1968.

Various projects are not the same and there are unequal delay penalties. The unequal delay penalties arise if the type supervision associated with each project is different, or the delay penalties are assigned by the customers, or if the profit contributions of the projects are different [11]. Since projects are different, a weighting factor is defined for each project to be able to produce objective functions in the formulation stage. The weighted factor can be calculated based on the delay penalties.

\section{SIMULATED ANNEALING ALGORITHM}

Simulated annealing is based on the similarity between solid annealing process and combinatorial optimization. The algorithm consists of several decreasing temperatures.

Suppose that finding the minimum of the cost function is favorable. Each temperature includes a sequence of iterations. First, the beginning temperature is chosen and the initial solution is selected and the cost function will be calculated. Then a new solution will be created in the neighborhood of the previous solution. New cost function will be calculated. If new cost function is less than the previous one, it will be accepted. If new cost function is less than the previous one, it will be accepted according to Metropolis's criterion [Metropolis et al., 1953] based on Boltzman's probability. According to Metropolis's criterion, if the difference between the cost function values of the current and the newly produced solutions $(\Delta \mathrm{E})$ is equal to or larger than zero, a random number $\delta$ in $[0,1]$ is generated from a uniform distribution and if

$$
\delta \leq e^{(-\Delta E / T)}
$$

Then the newly produced solution is accepted as the current solution. The number of new solutions which are created in each temperature is as many as the iteration number (termination condition). Iteration number can be a certain number of moves [10]. Then temperature will reduce upon temperature update rule and every above-said step will be iterated until the temperature goes down the minimum temperature (halting criteria) [9]. The result will be affected with the number of iterations and the speed of reducing temperature. The halting criterion in this research is equation (2).

Temperature $=T e^{(-r t)}$

Where $\mathrm{T}$ is the initial temperature, $\mathrm{r}$ is a cooling ratio, and $t$ is the number of times that temperature has been used. The chosen $r$ is 0.1 in this research.

\section{PROBLEM FORMULATION}

Assume there are two linear projects with $N_{1}+N_{2}$ activities, $\mathrm{M}$ locations, and I critical resources. $\mathrm{n}$ represents an activity i.e. one of $\left(N_{1}+N_{2}\right)$ activities but $n_{1}$ and $n_{2}$ represents activities of the first and second project respectively. The final program is based on this formulation. These formulas are based on those formulas which are proposed for a single linear project scheduling [10]. The problem is formulated as follows: 
Objectives:

$$
\begin{aligned}
& \text { MinimizeNa }\left\{w_{1} f\left(n_{1}, m\right)+w_{2} f\left(n_{2}, m\right)\right. \\
& \left.\mid n_{1}=1 . . N_{1} ; n_{2}=N_{1}+1 . . N_{1}+N_{2} ; m=1 . . M\right\} \\
& \text { Minimize } \sum_{t=1}^{T-1} \sum_{i=1}^{I} W_{i}\left(d p_{t, i}+d m_{t, i}\right)[10]
\end{aligned}
$$

Constraints:

- Activities precedence relationships

$$
\begin{aligned}
& s\left(n_{1}, m+1\right) \geq f\left(n_{1}, m\right) \\
& \forall n_{1}=1, N_{1} ; \forall m=1, M \\
& s\left(n_{2}, m+1\right) \geq f\left(n_{2}, m\right) \\
& \forall n_{2}=N_{1}+1, N_{1}+N_{2} ; \forall m=1, M \\
& s(1,1) \geq \alpha \\
& s\left(N_{1}+1,1\right) \geq \beta \\
& s\left(n_{1}, m\right) \geq f\left(p_{1}, m\right)+L\left(n_{1}, p_{1}\right) \\
& \forall m=1 \ldots M ; \forall p_{1} \in P_{1}(F S) \\
& s\left(n_{2}, m\right) \geq f\left(p_{2}, m\right)+L\left(n_{2}, p_{2}\right) \\
& \forall m=1 . . M ; \forall p_{2} \in P_{2}(F S) \\
& s\left(n_{1}, m\right) \geq s\left(p_{1}, m\right)+L\left(n_{1}, p_{1}\right) \\
& \forall m=1 \ldots M ; \forall p_{1} \in P_{1}(S S) \\
& s\left(n_{2}, m\right) \geq s\left(p_{2}, m\right)+L\left(n_{2}, p_{2}\right) \\
& \forall m=1 \ldots M ; \forall p_{2} \in P_{2}(S S) \\
& f\left(n_{1}, m\right) \geq s\left(p_{1}, m\right)+L\left(n_{1}, p_{1}\right) \\
& \forall m=1 . . M ; \forall p_{1} \in P_{1}(S F) \\
& f\left(n_{2}, m\right) \geq s\left(p_{2}, m\right)+L\left(n_{2}, p_{2}\right) \\
& \forall m=1 . . M ; \forall p_{2} \in P_{2}(S F) \\
& f\left(n_{1}, m\right) \geq f\left(p_{1}, m\right)+L\left(n_{1}, p_{1}\right) \\
& \forall m=1 . . M ; \forall p_{1} \in P_{1}(F F)
\end{aligned}
$$

$f\left(n_{2}, m\right) \geq f\left(p_{2}, m\right)+L\left(n_{2}, p_{2}\right)$

$\forall m=1 \ldots M ; \forall p_{2} \in P_{2}(F F)$

- $\quad$ Resource availability

$\sum_{m=1}^{M} \sum_{n=1}^{N_{1}+N_{2}} r_{i}(n, m, t) \leq R A_{i}(t)$

$\forall t=1, T ; \forall i=1, I$

- Activities completion

$\sum_{t=1}^{T} r_{i}(n, m, t) \geq T R_{i}(n, m)$

$\forall n=1, N_{1}+N_{2} ; m=1, M ; i=1, I$

$\sum_{n=1}^{N_{1}+N_{2}} \sum_{m=1}^{M}\left[r_{i}(n, m, t+1)-r_{i}(n, m, t)\right]-d p_{t, i}+d m_{t, i}=0$

$\forall t=1, T-1 ; i=1, I$

Notations:

$s\left(n_{i}, m_{i}\right)$ : Start time of activity $\mathrm{n}$ at location $\mathrm{m}$ in the first project

$f\left(n_{i}, m_{i}\right)$ : Finish time of activity $\mathrm{n}$ at location $\mathrm{m}$ in the second project

$d p_{t, i}$ : Absolute difference plus value of resource $\mathrm{I}$ assignment between day $\mathrm{t}+1$ and day $\mathrm{t}$

$d m_{t, i}:$ Absolute difference minus value of resource $\mathrm{I}$ assignment between day $\mathrm{t}+1$ and day $\mathrm{t}$ $W_{i}$ : Weighting factor for resource $\mathrm{i}$

$w_{i}$ : Weighting factor of various projects

$r_{i}(n, m, t)$ : Resource $\mathrm{i}$ assigned to activity $\mathrm{n}$ at location $\mathrm{m}$ at time $\mathrm{t}$

$R A_{i}(t)$ : Resource i availability at time $\mathrm{t}$

$T R_{i}(n, m)$ : Total amount of resource i required to complete activity $n$ at location $m$ $\alpha$ : Start time of the first project 
$\beta$ : Start time of the second project

$L(n, p)$ : The lead time between activity $\mathrm{n}$ and its predecessor $\mathrm{p}$

$p:$ Set of all predecessors of activity $\mathrm{n}$.

\section{SOLUTION METHODOLOGY}

A two-stage solution-finding procedure is introduced in this section. These two stages are explained briefly. In the first stage a simple feasible solution for each project is proposed. In this method we imagine that activities don't have any overlapping in each project so the first activity of each project in all locations is finished before the second activity of that with the second priority starts and it continues till each project is finished. In the other words, it is the maximum duration for each project but it guarantees that we have enough resources for activities to be done. It is assumed that resources are fixed for an activity in all locations in this stage.

Simulated annealing is used during the second stage to find the optimized solution. The number of one of the resources of one of the activities is changed in each iteration of the algorithm to find the best allocation. The problem will be solved by multiple-resource allocation algorithm. NTF concept is utilized in solving by considering multiple resource constraint. The resources assigned to a repetitive activity can be varied at different locations within a specified range. The goal is to find best assignment of resources to activities to have the minimum sum of weighted project durations and fluctuation of resources assigned for the projects. So search neighborhood is all possible resource assignments to activities. The maximum and minimum temperatures are 1000 and 1 respectively.

\section{NUMERICAL EXAMPLE}

In this section two projects are introduced as a multi-project problem to be solved by the model which is proposed. The following assumptions have been observed [3]:

1. A task can not be split. Once an activity is started, it will continue without interruption until it is finished.
2. Resources are limited. The limitation is assumed to be a constant across the entire project life span.

3. A resource con not be split, i.e., the resource amount is a positive integer.

4. Resources are assumed to maintain a constant productivity level within a certain range of assignment.

5. A resource can not be split.

Table 1 lists required information about the proposed artificial projects and their activities. Project 1 consists of activities A to $\mathrm{E}$ and project 2 consists of activities $\mathrm{F}$ to $\mathrm{K}$. Table 2 presents information about resources. It is assumed that resources are labors. The maximum number of Labor 1 and 2 are 8 and 6 respectively.

Table 1-Required Information About Activities

\begin{tabular}{|c|c|c|}
\hline Activity & Duration (Days) & Predecessor \\
\hline A & 4 & \\
\hline B & 2 & A (FS0) \\
\hline C & 2 & B(FS0) \\
\hline D & 2 & C(FS1) \\
\hline E & 1 & D(FS0) \\
\hline F & 3 & \\
\hline G & 2 & F (FS0) \\
\hline H & 4 & G (FS1) \\
\hline I & 3 & H (FS0) \\
\hline J & 1 & I (FS0) \\
\hline K & 4 & J(FS2) \\
\hline
\end{tabular}

The goal of this problem is to find the best resource assignment combination and the best sequence of activities to minimize the sum of weighted project durations and the fluctuation of resource usage. The main objective is to find the minimum of the sum and the second objective (minimum fluctuation of resource usage) is used when there are two schedules with the same sum. $\alpha$ and $\beta$ are both 0 in this problem. 
Table 2 Required Information About Resources

\begin{tabular}{|l|l|l|l|l|l|l|l|}
\hline $\begin{array}{l}\text { Ac } \\
\text {. }\end{array}$ & L1 & L2 & $\begin{array}{l}\text { Total } \\
\text { L1 } \\
\text { Require } \\
\text { d }\end{array}$ & $\begin{array}{l}\text { Total } \\
\text { Require } \\
\text { d }\end{array}$ & $\begin{array}{l}\text { Activity } \\
\text { priority }\end{array}$ & $\begin{array}{l}\text { L1 } \\
\text { Rang } \\
\text { e }\end{array}$ & $\begin{array}{l}\text { L2 } \\
\text { Rang } \\
\text { A }\end{array}$ \\
\hline B & 2 & 1 & $2 * 2=4$ & $2 * 1=2$ & 2 & $1-3$ & $1-2$ \\
\hline C & 2 & 1 & $2 * 2=4$ & $2 * 1=2$ & 3 & $1-3$ & $1-3$ \\
\hline D & 1 & 1 & $2 * 1=2$ & $2 * 1=2$ & 4 & $1-2$ & $1-2$ \\
\hline E & 1 & 1 & $1 * 1=1$ & $1 * 1=1$ & 5 & $1-2$ & $1-2$ \\
\hline F & 2 & 1 & $3 * 2=6$ & $3 * 1=3$ & 1 & $1-3$ & $1-2$ \\
\hline G & 1 & 2 & $2 * 1=2$ & $2 * 2=4$ & 2 & $1-2$ & $2-4$ \\
\hline H & 3 & 1 & $4 * 3=12$ & $4 * 1=4$ & 3 & $2-4$ & $1-3$ \\
\hline I & 1 & 1 & $3 * 1=3$ & $3 * 1=3$ & 4 & $1-2$ & $1-2$ \\
\hline J & 1 & 2 & $1 * 1=1$ & $1 * 2=2$ & 5 & $1-2$ & $1-3$ \\
\hline K & 2 & 1 & $4 * 2=8$ & $4 * 1=4$ & 6 & $2-4$ & $1-3$ \\
\hline
\end{tabular}

\section{RESULTS AND CONCLUSION}

The problem is written in Java programming language. The program is run 100 times. The average and the most optimized answer and other information which is achieved from 100 implementations are represented in table 3 . Table 4 shows the result of scheduling for some activities of the most optimized answer.

The following conclusions can be achieved from the results which are prepared.

- A large number of companies which are related to linear projects manage several projects simultaneously so these two concepts should be integrated in a single model.

- Multi-project and Linear scheduling with multiple resources can be integrated and solved simultaneously. This paper proposes a model to solve this problem.

- There are two approaches for scheduling of multi-project situations i.e. multiproject and single project approach. The multi-project approach is used in this paper.
- The model is verified by a successful numerical example. The summary of implementations is summarized in two tables.

Table 3Results of Implementations

\begin{tabular}{|l|l|l|l|}
\hline $\begin{array}{l}\text { No. } \\
\text { of } \\
\text { runs }\end{array}$ & $\begin{array}{l}\text { Most } \\
\text { Optimized } \\
\text { answer }\end{array}$ & Average & Variance \\
\hline 100 & 55 & 68.93 & 54.93 \\
\hline
\end{tabular}

Table 4 Result of Scheduling for Some Activities of the Most Optimized Answer

\begin{tabular}{|l|l|l|l|}
\hline Act. & Time & $\begin{array}{l}\text { L1 } \\
\text { Assigned }\end{array}$ & $\begin{array}{l}\text { L2 } \\
\text { Assigned }\end{array}$ \\
\hline A1 & $0-4$ & 3 & 2 \\
\hline A2 & $4-8$ & 3 & 2 \\
\hline A3 & $8-12$ & 3 & 2 \\
\hline G1 & $3-5$ & 1 & 2 \\
\hline G2 & $12-14$ & 1 & 2 \\
\hline G3 & $14-16$ & 1 & 2 \\
\hline
\end{tabular}

\section{REFERENCES}

[1] O'Brien, J.J. (1969) Scheduling Handbook. McGraw-Hill Book Company, New York.

[2] O’Brien, J.J. (1975) Vpm scheduling for highrise building. Journal of Construction. Div. ASCE, 101(Co4):895-905.

[3] Johnston, D.W. (1981) Linear scheduling method for highway construction. Journal of Construction Div. ASCE, 107(Co2):247-261.

[4] Peer, S. (1974) Network analysis and construction planning. Journal of Construction Engineering and Management, ASCE, 100(Co3):203-210.

[5] Harmelink, D.J. (1995) Linear scheduling model: the development of a linear scheduling model with micro computer applications of 
highway construction control. $\mathrm{PhD}$ thesis, Iowa State University, Ames, Iowa.

[6] Mattila, K.G. (1997) Resource Leveling of Linear Schedules: A Mathematical Approach Using Integer Linear Programming. PhD thesis, Purdue University.

[7] Liu, S.S. (1999) Resource-Constrained Scheduling of Linear Projects: A Heuristic Approach Using Tabu Search Heuristics. PhD thesis, Purdue University.

[8] Leu, S.S. and Hwang, S.T. (2001) Optimal repetitive scheduling model with Shareable resource constraint. Journal of Construction
Engineering and Management, ASCE, 127(4):270-280.

[9] Kirkpatrick, S., Gelatt, C.D., and Vecchi, M.P. (1983) Optimization by simulated annealing. Science, 220:671-680.

[10]Yen, C.I. (2005) Simulated annealing for optimizing linear scheduling projects with multiple resource constraints. $\mathrm{PhD}$ thesis, Purdue University.

[11] Kurtulus, I. (1985) Multiproject Scheduling: Analysis of Scheduling Strategies Under Unequal Delay Penalties. Journal of Operations Management, Vol. 5, No. 3. 